\title{
Los programas de Interculturalidad. Una construcción desde sus actores
}

\author{
Esparza de la O María Teresa \\ Universidad Pedagógica Nacional del Estado de Chihuahua, Unidad Chihuahua \\ Vega Villarreal Sandra \\ Universidad Pedagógica Nacional del Estado de Chihuahua, Unidad Chihuahua \\ svegavillarreal@gmail.com
}

\section{Resumen}

El presente documento expone de manera sintética los resultados la primera etapa de una investigación - acción que se está desarrollando con profesores del medio indígena en la sierra tarahumara. La etapa centró su atención en cómo enfrentar los retos de la interculturalidad que exige el nuevo contexto social a partir del desarrollo de programas educativos que coadyuven a resolver la problemática concreta que representa el bilingüismo la cual, se identificó como la problemática principal que enfrentan los actores educativos, así como la comunidad. El documento describe cómo se llevó a cabo la construcción de las acciones pedagógicas, las formas de evaluar y cómo se replantearon los proyectos generados por la colectividad.

\section{Palabras clave}

Interculturalidad, programas educativos, bilingüismo, investigación-acción.

\section{Introducción}

El presente trabajo evidencia de manera sintética los resultados de una investigación acción realizada con profesores de educación indígena en la sierra de Chihuahua. El estudio se coordina con catedráticos de la Universidad Pedagógica Nacional y estudiantes de la maestría en interculturalidad que ofrece la institución. Por tanto, el contexto de trabajo son comunidades serranas donde los estudiantes desarrollan su función como docentes, todas ellas del municipio de Bocoyna.

El esfuerzo por desarrollar este tipo de investigación surge de la necesidad apremiante de transformar la realidad de los profesores, alumnos y comunidad, poniendo en primer término la falta de comunicación por la predominancia de la población monolingüe entre los involucrados. Este problema se vuelve más urgente ante las exigencias que la Educación Básica demanda de los profesores con la Reforma Educativa a nivel Nacional en el cual se presenta la reformulación y adecuación de la realidad multi e intercultural de la población serrana.

La metodología utilizada en este estudio es la investigación acción, pues busca el conocimiento y transformación de una realidad concreta, permite la adquisición del conocimiento colectivo. Combina la investigación social, el trabajo educativo y la 
acción. Tiene alcances comunitarios y es precisamente la comunidad quien controla el proceso global de investigación (Rodríguez, 2002).

\section{Objeto de estudio}

Aunque de manera oficial se han conformado proyectos y programas interinstitucionales para la transformación circular de la formación docente que define el perfil de maestro en educación primaria intercultural bilingüe, matrices circulares por asignatura, plan de estudios, no se atiende el problema de fondo, pues los involucrados en la comunidad permanecen al margen del diseño y operación de estos proyectos, los cuales se implementan Así mismo cabe reflexionar en que existen comunidades donde por las características geográficas, climáticas, sociales, económicas y culturales, así como el problema de la comunicación y el transporte, el acceso a la educación en los municipios y comunidades rurales es precario. Muchas veces esta inaccesibilidad acompañada de la carencia de plazas y salarios para contratar nuevos maestros., además de la falta de condiciones mínimas de infraestructura y equipamiento.

Ante este complejo contexto con tantas necesidades apremiantes de atender y con una realidad tan adversa, surgen de manera colectiva las siguientes preguntas de investigación:

¿Cómo desarrollar una educación intercultural que atienda los problemas de bilingüismo?

¿Qué proyectos pedagógicos pueden generar la transformación de la realidad educativa indígena a partir del uso de la lengua?

¿Cómo evaluar y reorientar los proyectos generados?

El objetivo principal de la presente investigación fue transformar la comunicación entre profesores, alumnos y 20 comunidades indígenas a partir del bilingüismo. Mismo que se considera una etapa de la transformación de la realidad que viven estos agentes educativos.

\section{Referentes teóricos}

Es de suma importancia reconocer que el concepto intercultural no solo se limita a la población indígena, sino que es un concepto en construcción que cada día todos los involucrados debemos pensar en la otredad. La interculturalidad requiere análisis rigurosos que favorezcan la comprensión de los conflictos que necesariamente surgen en este contexto (riesgo de asimilación de pérdida de identidad cultural, de marginación social entre otros.) y que aporten elementos para definir políticas críticas capaces de enfrentar lo que constituyen los auténticos obstáculos en este camino: la injusticia y la desigualdad.

La inspiración de los pueblos originarios indígenas en el resto de la región latinoamericana y de las comunidades étnicas, tiene que ver con los derechos del medio ambiente y con el manejo racional del mismo, de modo que tales recursos sirvan para generar empleo y para satisfacer necesidades de vida. Se busca entonces, elementos de análisis que permitan plantear pedagógicamente la vinculación entre los manejos territoriales y medio ambiente en el marco de los principios éticos referidos a la salvaguarda de toda forma de vida y de la biodiversidad. Es importante la previsión de la investigación como un recurso metodológico y pedagógico más allá de los textos escolares. Estos materiales de apoyo deberán ser construidos con el propósito de promover el autoaprendizaje sustentándose en fundamentos y principios del aprendizaje infantil del niño en su propia región y en su propia etnia.

Este trabajo describe la importante investigar los procesos de estandarización de las normas escritas de las lenguas indígenas y 
etnias sin olvidar el reto que significa el desarrollo de las metodologías de enseñanza de las segundas lenguas y por supuesto la capacitación de los docentes en el uso de las mismas, pero desde la construcción de los actores.

\section{Discusión de resultados}

La primera fase de la investigación acción desarrollada fue diagnosticar de manera colectiva la problemática concreta de la comunidad (Yopo, 1999). El centro de la discusión en los diferentes círculos de cultura giró en torno a la importancia de llevar a la acción y proyectos comunitarios promoviendo así una cultura educativa, que facilite la integración de toda población en función de proyectos colectivos que vayan más allá del aula escolar. Estos proyectos incluyen la formación y conocimiento de sus derechos, $y$ es necesario orientar a la comunidad educativa en la evaluación de las prácticas educativas, pedagógicas y de aprovechamiento escolar, una evaluación orientada al mejoramiento de los procesos educativos con el propósito de contribuir a la toma de decisiones, y no sujetos a requisitos administrativos tomados con criterios ajenos a procesos educativos comunitarios.

En la segunda fase de la investigación consistente en la priorización de las necesidades comunitarias (De Shutter, 1998), se definió como prioridad el diseño de acciones de formación docente y de producción de materiales en el ámbito de la enseñanza de una primera lengua teniendo un puente a la enseñanza de una segunda lengua en el marco del bilingüismo y de la interculturalidad.

En la fase de acción pedagógica, se diseñó y se planeó la implementación de un proyecto educativo que centra su interés en la escritura, intereses que lleva varios años y el foco no solo a la producción escrita sino a la indagación sobre las condiciones educativas que brindan apoyo o no al desarrollo de la escritura, y lo más relevante es la práctica de la enseñanza de la escritura en el contexto bilingüe. Clay (1983) como la escritura crea una representación permanente de significados por lo que esto conlleva la importancia de que el maestro construya su propia metodología de trabajo en 1er y 2 do Grado partiendo de los intereses del niño y del conocimiento de su realidad, creando así textos que le den sentido y significado a el niño que realiza un proceso de aprendizaje.

En base a las investigaciones se afirma que la lengua materna plenamente desarrollada proporciona la base lingüística para el desarrollo del lenguaje y la alfabetización. Como (Cummins, 1994) que la instrucción de la lengua materna permite a los alumnos a trabajar a su propio nivel cognitivo. Se ha señalado la importancia de la alfabetización de la lengua materna, así como una segunda lengua aun cuando ambos tienen sistemas de escritura distintos (Cummins, 1994) y se han encontrado como resultados cuando la alfabetización de la lengua inicia primero que cuando se trabaja simultáneamente con la segunda. Motivo por el cual este trabajo manifiesta el avance de un proceso de investigación donde se pretende que el maestro-alumno LEPEPMI'90, sea capaz de construir su propia metodología regional partiendo de la realidad del niño en la elaboración de materiales que permitan el uso y función de textos con significado para el niño raramuri, pima, tepehuan, partiendo de su región o comunidad. El saber leer y escribir en la lengua materna facilita el aprendizaje de una segunda lengua, por la transferencia de conocimientos y procesos de aprendizaje (Collier, 1992).

Las estrategias que componen el proyecto se fueron aplicando, evaluando y reorientando, en los casos que se consideró pertinente. En cada una de las estrategias se puso como centro el desarrollo de los procesos de aprendizaje situados en la educación indígena. En las actividades se 
incorporaron elementos comunes para toda la educación básica del medio indígena. Sin embargo, un aspecto nodal fue reconocer de manera explícita los valores, los modos de vida y la diversidad cultural que confluye en la comunidad para el diseño de cada actividad.

Bajo los preceptos anteriores la acción pedagógica abarca una serie de acciones. La primera de ellas fue la construcción de códigos comunicativos entre la comunidad y la escuela. Esto se llevó a cabo a través de la confluencia de lenguas y la construcción de significados comunes a partir de los significantes concretos de la comunidad y la escuela. Todo este trabajo de meses de intercambio lingüístico permitió la elaboración de un diccionario bilingüe, que en una propuesta inicial fue elaborado por los profesores y los alumnos y corregido y redefinido por la comunidad, hasta que se obtuvo el producto deseado.

Otra de las estrategias que constituyeron este proyecto fue la "historia sin voz" la cual consiste en la reconstrucción de la historia de la comunidad desde una visión compartida entre dos culturas. Al hacer esto se promueve el análisis y la reflexión sobre las cosmovisiones culturales que convergen en un mismo espacio. Se debatieron en círculos de cultura (Freire, 1999) las cuestiones culturales que permiten la convivencia a través del bilingüismo.

Dentro de la acción pedagógica también se realizaron una serie de actividades culturales bajo la diada escuela - comunidad. Una muy importante fue la construcción colectiva de cuentos raramuris. Éstos se elaboraron originalmente en la lengua materna de los diferentes actores y entre todos los fueron transformando a la lengua de los otros, de tal manera que los cuentos que quedaron al final fueron aquellos que la comunidad consensó en significado e hizo suyos como una integración cultural.
Una acción muy parecida fue el de cantos y juegos raramuris, donde con la participación de todos se logró recopilar una serie de cantos y juegos de la comunidad que se trabajaron $d$ la misma manera de los cuentos. Esto mostró que el debate y el consenso son la forma de interculturalidad más genuina para la transformación cultural.

Finalmente y como estrategia más importante se desarrolló entre estudiantes y maestros un proyecto pedagógico para trabajarse dentro del aula. Este proyecto consistió en construir una metodología colectiva, que les permitiera acceder a los procesos de lecto-escritura. Esta fase implicó algunos meses de debate sobre los significados lingüísticos y culturales para el desarrollo de los contenidos de aprendizaje del castellano y del raramuri, así es que tuvieron que generarse una serie de recursos, aparte del directorio existente que les estuvieran apoyando de manera permanente en ese debate. Una de las características centrales de la metodología construida es que establece prioridades en lo que desde su cultura, los estudiantes necesitan aprender. La diseñan desde su cultura, su experiencia de vida y utilizan sus saberes para transformar su realidad más allá de la escuela.

\section{Conclusiones}

La investigación acción es un proceso permanente y continuo de educación para la reflexión y la acción, por lo que no termina con la conclusión de un proyecto de acción pedagógica, sino que se establece como forma de vida de la comunidad y la escuela.

El proyecto desarrollado en esta primera etapa muestra que cuando los programas educativos surgen de los involucrados en la problemática tienen un gran impacto de transformación, a diferencia de los que implementan actores ajenos al contexto. 
Con respecto al bilingüismo, este proyecto demostró que más allá de enseñar una segunda lengua, se promovió el encuentro intercultural donde dos o más culturas se encuentran, se identifican, debaten y consensan formas de convivencia, sin que haya una invasión cultural, sino un proceso dialógico y educativo genuino.

\section{Referencias}

COLLIER, Jane, 1992. Programa Nacional de la medicina indígena tradicional. México, UNAM.

CUMMINS, Jim, 1994. ¿Qué sabemos de la educación bilingüe? Perspectivas psicolingüísticas y sociológicas. Revista de Educación No. 326 "Educación y bilingüismo". España, Ministerio de Educación.

FREIRE, Paulo, 1999. Extensión o comunicación, México. Siglo XXI editores.

DE SHUTTER, Anton, 1998. Método y proceso de la investigación-acción en la capacitación rural. México, cuadernos CREFAL.

YOPO, Boris, 1999. Metodología de la investigación participativa. México, Cuadernos CREFAL. 
RECIE. Revista Electrónica Científica de Investigación Educativa Vol. 1, núm. 2, enero-diciembre 2013, pp. 367-371. 